\title{
Analytical Solutions for Contaminant Diffusion in Double-Layered Porous Media
}

\author{
Yu-Chao Li ${ }^{1}$ and Peter John Cleall ${ }^{2}$
}

\begin{abstract}
Analytical solutions for conservative solute diffusion in one-dimensional double-layered porous media are presented in this paper. These solutions are applicable to various combinations of fixed solute concentration and zero-flux boundary conditions (BC) applied at each end of a finite one-dimensional domain and can consider arbitrary initial solute concentration distributions throughout the media. Several analytical solutions based on several initial and BCs are presented based on typical contaminant transport problems found in geoenvironmental engineering including (1) leachate diffusion in a compacted clay liner (CCL) and an underlying stratum; (2) contaminant removal from soil layers; and (3) contaminant diffusion in a capping layer and underlying contaminated sediments. The analytical solutions are verified against numerical solutions from a finite-element method based model. Problems related to leachate transport in a CCL and an underlying stratum of a landfill and contaminant transport through a capping layer over contaminated sediments are then investigated, and the suitable definition of the average degree of diffusion is considered.
\end{abstract}

DOI: 10.1061/(ASCE)GT.1943-5606.0000365

CE Database subject headings: Analytical techniques; Containment; Diffusion; Layered systems; Porous media.

Author keywords: Analytical techniques; Containment; Diffusion; Layered systems; Porous media.

\section{Introduction}

Contaminant transport analysis is undertaken when considering geoenvironmental problems such as the evaluation and design of engineered/natural containment barrier systems for waste disposal and the containment and remediation of existing contaminated soils (Sharma and Reddy 2004). In particular, contaminant diffusion analysis in porous media is of great importance in that diffusion often dominates the contaminant transport processes in engineered barriers, natural containment systems, and geological soil/rock media (Shackelford and Lee 2005).

One-dimensional diffusion of solute in a semiinfinite or finite homogeneous porous medium can be analyzed via use of analytical solutions for diffusion in solids (Crank 1956; Carslaw and Jaeger 1959). Contaminant transport in a system consisting of two soil layers, whose soil and transport properties are quite different from each other, is often observed in geoenvironmental engineering problems. For example, leachate transfer through a compacted clay liner (CCL) and an underlying stratum in landfills (Rowe et al. 2004) and contaminant diffusion in capping layers over contaminated sediments (Palermo et al. 1998b; Lampert and

${ }^{1}$ Lecturer, MOE Key Laboratory of Soft Soils and Geoenvironmental Engineering, Department of Civil Engineering, Zhejiang Univ., Hangzhou 310058, China; formerly, Research Associate, Geoenvironmental Research Centre, Cardiff School of Engineering, Cardiff Univ., Cardiff CF24 3AA, Wales, U.K. E-mail: yuchao_li@hotmail.com

${ }^{2}$ Lecturer, Geoenvironmental Research Centre, Cardiff School of Engineering, Cardiff Univ., Cardiff CF24 3AA, Wales, U.K. (corresponding author). E-mail: cleall@cardiff.ac.uk

Note. This manuscript was submitted on April 15, 2009; approved on April 6, 2010; published online on April 10, 2010. Discussion period open until April 1, 2011; separate discussions must be submitted for individual papers. This paper is part of the Journal of Geotechnical and Geoenvironmental Engineering, Vol. 136, No. 11, November 1, 2010. CASCE, ISSN 1090-0241/2010/11-1542-1554/\$25.00.
Reible 2009) are often considered. These two examples are illustrated in Fig. 1. The range of initial and boundary conditions (BCs) of many solutions is limited and is not suitable for many practical contaminant diffusion problems. Some work has been done on solving the diffusion or advection-dispersion equation of solute transports in two- or multilayered porous media using the Laplace transform method (Leij et al. 1991; Leij and van Genuchten 1995), the integral transform method (Liu et al. 1998), and an approach combining the Laplace transformation method and binomial theorem (Liu and Ball 1998). Also, an analytical solution for contaminant diffusion through multilayered system was presented by Chen et al. (2009); however, only scenarios with a fixed top BC are considered as contaminant diffusion in landfill liners is the main focus of their work. The form of the solutions considering multilayered system above (Chen et al. 2009; Leij et al. 1991; Leij and van Genuchten 1995; Liu and Ball 1998; Liu et al. 1998) while able to yield valuable insights into the mechanisms occurring is relatively complex and is not readily amenable to simple implementation.

This paper presents analytical solutions for conservative solute (in terms of mass) diffusion in one-dimensional double-layered porous media subjected to arbitrary initial and BCs. The term "conservative solute" is used here to mean that no loss in mass of the solute species occurs during transport as opposed to indicating a nonreactive solute. In fact, the solute is considered to be reactive via consideration of reversible, linear, and instantaneous sorption by the use of a retardation factor. This work is inspired by the analogy between Terzaghi's governing equation for consolidation and Fick's governing equation for diffusion presented by Shackelford and Lee (2005). The governing equations of solute diffusion are solved following the approach of Lee et al. (1992) and Xie (1994) who considered consolidation in doublelayered soils. The novelty of the solutions presented herein is both in terms of the simplicity of the solutions relative to Laplace transform approaches and the wider range of $\mathrm{BCs}$ that are considered. In contrast to Chen et al. (2009), the number of layers is 


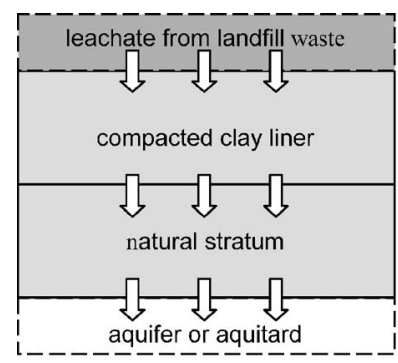

(a)

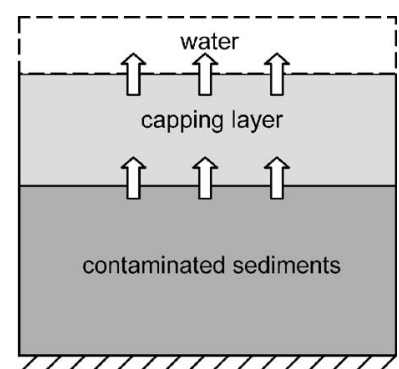

(b)
Fig. 1. Examples of contaminant diffusion in double-layered soils: (a) contaminant transport in a compacted clay landfill liner and an underlying stratum; (b) contaminant diffusion through a capping layer over contaminated sediments

restricted to 2 ; this restriction has the advantage of the resulting eigenvalued function being considerably less complex. Several analytical solutions subjected to a series of initial and BCs are presented based on typical contaminant transport problems found in geoenvironmental engineering. The analytical solutions are verified against numerical solutions by consideration of a hypothetical diffusion problem in a double-layered system and applied to analyze both leachate transport in a CCL of a landfill and an underlying stratum and contaminant transport in capped contaminated sediments.

\section{Theory}

\section{Problem Formulation}

A porous medium consisting of two individual homogeneous layers is considered, as illustrated in Fig. 2. A coordinate system $(z)$, whose positive direction is downward, is adopted, and the top of the upper layer is chosen as the origin of $z$. Each layer has its own constant effective diffusion coefficient $\left(D_{i}^{*}\right)$, retardation factor $\left(R_{d i}\right)$, and porosity $\left(n_{i}\right)$. The subscript $i$ represents the layer with $i=1$ corresponding to the upper layer and $i=2$ to the lower layer.

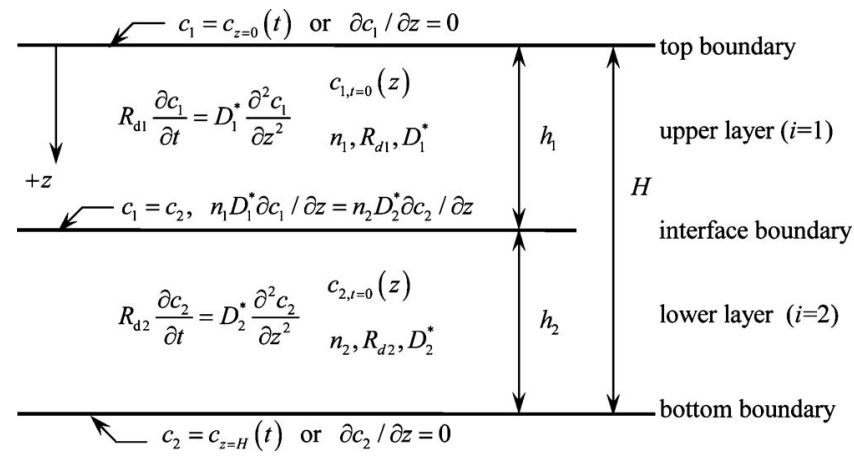

Fig. 2. Schematic representation of generalized domain for solute diffusion in a double-layered porous medium
The thicknesses of the upper layer and the lower layer are $h_{1}$ and $h_{2}$, respectively, and the total thickness $H$ is $h_{1}+h_{2}$.

The effective diffusion coefficient is defined as the product of the apparent tortuosity factor $\tau_{a}$ and the aqueous diffusion coefficient for the solutes $D_{0}$, i.e., $D^{*}=\tau_{a} D_{0}$ (Freeze and Cherry 1979; Shackelford and Daniel 1991). A linear reversible sorption isotherm (Freeze and Cherry 1979) is considered such that the retardation factor can be written as $R_{d i}=1+\rho_{i} K_{d i} / n_{i}$, where $\rho_{i}=$ bulk dry density of soil and $K_{d i}=$ distribution coefficient. The governing equation of solute diffusion in each layer (Shackelford and Daniel 1991) can be expressed as follows:

$$
R_{d i} \frac{\partial c_{i}}{\partial t}=D_{i}^{*} \frac{\partial^{2} c_{i}}{\partial z^{2}} \quad(i=1,2)
$$

where $t$ represents time and $c_{i}=$ solute concentration in the pore water of the $i$ th layer.

The initial condition for the problem considered can be expressed as

$$
\left.c_{i}\right|_{t=0}=c_{i, t=0}(z) \quad(i=1,2)
$$

where $c_{i, t=0}(z)=$ arbitrary function for the initial solute concentration distribution in the $i$ th soil layer. Several combinations of BCs are considered, as shown in Table 1 , where $c_{z=0}(t)$ and $c_{z=H}(t)$ $=$ time-dependent functions for the boundary solute concentration at the top and bottom, respectively.

Solute concentration continuity and flux continuity are satisfied at the interface between layers as follows:

$$
\left.c_{1}\right|_{z=h_{1}}=\left.c_{2}\right|_{z=h_{1}}
$$

$$
\left.n_{1} D_{1}^{*} \frac{\partial c_{1}}{\partial z}\right|_{z=h_{1}}=\left.n_{2} D_{2}^{*} \frac{\partial c_{2}}{\partial z}\right|_{z=h_{1}}
$$

Following Shackelford and Lee (2005), the relative amount of solute removed or gained in the two layers at any elapsed time can be represented by the average degree of diffusion, $U_{c}(t)$, as

$$
U_{c}(t)=\frac{M(0)-M(t)}{M(0)-M(\infty)}
$$

where $M(t)=$ solute mass per unit area within the double-layered system at time $t$ and can be expressed by

$$
M(t)=n_{1} R_{d 1} \int_{0}^{h_{1}} c_{1}(z, t) d z+n_{2} R_{d 2} \int_{h_{1}}^{H} c_{2}(z, t) d z
$$

The definition of the average degree of diffusion in Eq. (4) is similar to the average degree of consolidation in Terzaghi's theory of consolidation (Terzaghi 1943). However, the solute mass in soil, rather than the solute mass in pore water as used by Chen et al. (2009), is considered in Eq. (4). This difference in definition of average degree of diffusion is discussed later in the "Applications" section.

The diffusive solute mass flux in soil, $J_{c}$, can be written in accordance with Fick's first law for diffusion in soil as follows (Shackelford 1991; Shackelford and Lee 2005):

Table 1. BCs for Double-Layered Systems Considered

\begin{tabular}{lcr}
\hline Boundary & Fixed concentration (Dirichlet) BC & Zero-flux (von Neumann) BC \\
\hline Top of layered system & $\left.c_{1}\right|_{z=0}=c_{z=0}(t)$ & $\partial c_{1} /\left.\partial z\right|_{z=0}=0$ \\
Bottom of layered system & $\left.c_{2}\right|_{z=H}=c_{z=H}(t)$ & $\partial c_{2} /\left.\partial z\right|_{z=H}=0$ \\
\hline
\end{tabular}




$$
J_{c}(z, t)=-n_{i} D_{i}^{*} \frac{\partial c_{i}(z, t)}{\partial z}
$$

Therefore, $J_{c}$ may be obtained at any spatial or temporal location via substitution of the spatial derivative of the solute concentration from the analytical solution.

\section{Analytical Solutions}

Analytical solutions for the governing equations, i.e., Eq. (1), subjected to the initial condition, i.e., Eq. (2), and the BCs in Table 1 and Eq. (3) are presented in this section. These are developed following the approach proposed by Lee et al. (1992) and Xie (1994) who considered the problem of linear elastic smallstrain consolidation in double-layered soils. Although Fick's governing equation for diffusion is analogous to Terzaghi's governing equation for consolidation (Shackelford and Lee 2005), the flux continuity condition at the interface of layers is different from that of consolidation as a result of both its porosity dependence, i.e., Eq. (3b), and being based on Fick's first law for diffusion versus Darcy's law for consolidation.

The following dimensionless parameters for the soil and transport properties are defined to simplify the formulations of the solutions:

$$
\delta=\frac{D_{2}^{*}}{D_{1}^{*}} ; \quad \rho=\frac{R_{d 2}}{R_{d 1}} ; \quad \nu=\frac{n_{2}}{n_{1}} ; \quad \theta=\frac{h_{2}}{h_{1}}
$$

\section{Fixed Surface - Zero-Flux Base Scenario}

A scenario with a fixed, time-dependent concentration BC at the top and a zero-flux BC at the bottom (i.e., fixed surface - zero-flux base scenario), as given in Table 1, is considered in this section. A solution for consolidation of a clay layer with free-draining BCs at both the top and bottom boundaries can be expressed as the sum of an infinite series of the product of a coefficient, a sinusoidal function, and an exponential function, representing the initial excess pore water pressure, depth, and elapsed time, respectively (Terzaghi 1943). Following the form of the solutions for such consolidation problems presented by Terzaghi (1943) and Lee et al. (1992), the solution for the scenario considered can be written as follows:

$$
\begin{gathered}
c_{i}(z, t)=c_{z=0}(t)+\sum_{m=1}^{\infty}\left[B_{m}+C_{m} S_{m}(t)\right] g_{m i}(z) \exp \left(-\beta_{m} t\right) \\
g_{m 1}(z)=\sin \left(\lambda_{m} \frac{z}{h_{1}}\right) \\
g_{m 2}(z)=A_{m} \cos \left(\mu \lambda_{m} \frac{H-z}{h_{1}}\right)
\end{gathered}
$$

where $A_{m}, B_{m}, C_{m}, \beta_{m}, \lambda_{m}$, and $\mu=$ coefficients to be determined and $S_{m}(t)=$ function with respect to time $t$ and to be determined [which is introduced due to the term of the time-dependent concentration $\mathrm{BC}$ at the top, i.e., $\left.c_{z=0}(t)\right]$. Note that the function $S_{m}(t)=0$ if the fixed concentration BC is time independent.

The types of the trigonometric functions in Eqs. (8b) and (8c) are chosen according to the BCs applied at the top and the bottom of the system, respectively, with a sine function chosen for the fixed $\mathrm{BC}$ and a cosine function chosen for zero-flux BCs. The value of the series function term in Eq. $(8 a)$ equals to zero at the top (that is, $z=0)$ using Eq. $(8 b)$ and the value of the term of the series function's derivative with respect to $z$ in Eq. (8a) equals to zero at the bottom (that is, $z=H$ ) using Eq. $(8 c)$. Consequently, the BCs at both the top and the bottom for the scenario considered here can be satisfied by the solution form of Eqs. $(8 a)-(8 c)$.

Substitution of Eqs. $(8 a)-(8 c)$ into Eqs. $(3 a)$ and $(3 b)$, i.e., to consider continuity at the interface between the layers, yields

$$
A_{m}=\sin \lambda_{m} / \cos \left(\mu \theta \lambda_{m}\right)
$$

$$
\delta \nu \mu \sin \lambda_{m} \sin \left(\mu \theta \lambda_{m}\right)-\cos \lambda_{m} \cos \left(\mu \theta \lambda_{m}\right)=0
$$

where Eq. (10) =eigenvalued function of $\lambda_{m}$. Further substitution of Eqs. ( $8 a)$ and (8b) into Eq. (1), to consider the solute diffusion within the upper layer, yields

$$
\begin{gathered}
\beta_{m}=\frac{D_{1}^{*}}{R_{d 1}} \frac{\lambda_{m}^{2}}{h_{1}^{2}} \\
S_{m}(t)=\int_{0}^{t} \frac{d c_{z=0}(\tau)}{d \tau} \exp \left(\beta_{m} \tau\right) d \tau \\
\sum_{m=1}^{\infty} C_{m} g_{m 1}=-1
\end{gathered}
$$

Similarly, substitution of Eqs. (8a) and (8c) into Eq. (1), to consider the solute diffusion within the lower layer, yields

$$
\begin{gathered}
\mu=\sqrt{\rho / \delta} \\
\sum_{m=1}^{\infty} C_{m} g_{m 2}=-1
\end{gathered}
$$

Finally, substitution of Eqs. (8a)-(8c) into Eq. (2), to consider the initial conditions, yields

$$
c_{i, z=0}(0)+\sum_{m=1}^{\infty} B_{m} g_{m i}(z)=c_{i, t=0}(z) \quad(i=1,2)
$$

Using the following orthogonal relations:

$$
\begin{gathered}
\int_{0}^{h_{1}} g_{m 1}(z) g_{n 1}(z) d z+\rho v \int_{h_{1}}^{H} g_{m 2}(z) g_{n 2}(z) d z \\
\quad=\left\{\begin{array}{cc}
0 & m \neq n \\
\frac{1}{2} h_{1}\left(1+\rho \nu \theta A_{m}^{2}\right) & m=n
\end{array}\right.
\end{gathered}
$$

the following formulations for $B_{m}$ and $C_{m}$ based on Eqs. (13) and (15)-(17) can be obtained:

$$
\begin{gathered}
B_{m}=2 \frac{\int_{0}^{h_{1}} c_{1, t=0}(z) g_{m 1}(z) d z+\rho \nu \int_{h_{1}}^{H} c_{2, t=0}(z) g_{m 2}(z) d z}{h_{1}\left(1+\rho \nu \theta A_{m}^{2}\right)} \\
-c_{z=0}(0) \frac{2}{\lambda_{m}\left(1+\rho \nu \theta A_{m}^{2}\right)} \\
C_{m}=-\frac{2}{\lambda_{m}\left(1+\rho \nu \theta A_{m}^{2}\right)}
\end{gathered}
$$

The integral terms in Eq. (18) can be written explicitly if the initial concentration function, $c_{i, t=0}(z)$, has a simple form, such as a unique function; otherwise numerical integration techniques (Chapra and Canale 2006) can be employed to solve the integral. 
The analytical solution presented in this section is summarized in Table 2 as Scenario 2.

\section{Other Boundary Scenarios}

Three other scenarios can be considered based on the other possible combinations of different BCs at the top and the bottom of the system, as indicated in Table 2. Similar solutions to that for the fixed surface and zero-flux base scenario can be established for these three scenarios and are also detailed in Table 2. Sine functions again are adopted in the expressions of $g_{m 1}(z)$ and $g_{m 2}(z)$ for the fixed concentration BC, whereas cosine functions are adopted for the zero-flux BCs. The unknown coefficients and functions can be obtained following the procedure presented for the fixed surface-zero-flux base scenario and are also listed in Table 2.

The dichotomy technique, recommended by Xie (1994), is adopted to solve the type of eigenvalue function of $\lambda_{m}$ presented in the analytical solutions. However, simple formulations of $\lambda_{m}$ can be obtained if $\delta \nu \mu=1$, that is, $\nu \sqrt{\delta \rho}=1$, as follows:

$$
\lambda_{m}=\left\{\begin{array}{cc}
\frac{m \pi}{1+\mu \theta} & (\text { Scenarios } 1 \text { and } 4) \\
\frac{(2 m-1) \pi}{2(1+\mu \theta)} & (\text { Scenarios } 2 \text { and } 3)
\end{array}\right.
$$

With various combinations of initial and BCs, the analytical solutions presented above can be utilized to analyze many typical cases for solute diffusion problems existing in geoenvironmental engineering. A number of possible cases are considered herein, and the solutions are listed in Table 3 . These solutions can be used to consider the following solute transport problems:

1. Leachate diffusion in a CCL and an underlying stratum (Cases A and D);

2. Contaminant removal from soil layers (Cases B and E); and 3. Contaminant diffusion in a capping layer and underlying contaminated sediments (Cases C, F, and G).

A constant, fixed concentration BC is included in Cases A-F, which enables the analytical solutions to have a simple form, compared to those for the general scenarios, as listed in Table 2. The formulations of average degree of diffusion for the cases considered are also given in Table 3. Nonstandard cases, including time-dependent BCs and complex initial conditions, may be analyzed on the basis of superposition (Taylor 1948; Shackelford and Lee 2005).

In this paper, convergence of determination of the series functions in the formulations for the solute concentration and the average degree of diffusion is regarded to have been achieved when the value of the final series term considered is less than $1 \times 10^{-8}$. Based on an investigation of the solutions presented herein, satisfaction of this criterion usually requires less than 30 series terms for an accurate solution.

\section{Verification}

The analytical solutions herein are verified via consideration of a hypothetical diffusion problem in a double-layered system. The results obtained from the analytical solutions are compared with those from a numerical solution using the finite-element method (Cleall et al. 2007; Seetharam et al. 2007).

The two layers are defined to initially have zero solute concentrations. The solute concentration is assumed to be constant at the boundaries, with a value of $c_{0}$ at the top boundary and a zero concentration at the bottom boundary, corresponding to Case A in Table 3. The soil and transport properties for the upper layer are assumed as $D_{1}^{*}=5 \times 10^{-10} \mathrm{~m}^{2} / \mathrm{s}, R_{d 1}=2.0$, and $n_{1}=0.4$. A number of analyses have been undertaken with varying values of $\delta, \rho, \nu$, $\theta$, and $H$ to illustrate the uniqueness of the solutions and results of these analyses follow.

First, three series of analyses are performed, all with $H$ $=1.0 \mathrm{~m}$ and $\theta=1$, where the value of one of the parameters given by Eq. (7) is varied while the other parameters are maintained at unity. Calculated solute concentration profiles at 10 years for these three series of analyses are shown in Figs. 3(a-c), respectively, together with those obtained by the numerical approach. The impact of the variation in the effective diffusion coefficients and the porosities between the two layers can be clearly seen with a distinct change in the concentration gradient (i.e., shape of concentration profile) at the interface from Figs. 3(a and c). This result is due to the fact that the interface BCs applied are dependent on the effective diffusion coefficients and the porosities [see Eq. $(3 b)$ ]. Such concentration gradient changes are not apparent in Fig. 3(b) because the continuity in mass flux at the interface is independent of retardation.

Solute concentration profiles at 2, 5, 10, and 20 years and for the long term, for a case having $\delta=\rho=\nu=0.5, \theta=1$, and $H$ $=1.0 \mathrm{~m}$, are presented in Fig. 3(d), and the solute penetration process can be seen clearly. The impact of the variation in effective diffusion coefficients and the porosities between the two layers can be seen at the interface in all but the 2-year profile because, at that short elapsed time, the solute has not yet reached the interface in any significant quanity.

Finally, a series of cases with $\delta=\rho=\nu=0.5$ and varying total thicknesses (i.e., $H=0.5,1.0,2.0 \mathrm{~m}$ ) and different values of the ratios of thickness for the lower layer relative to the upper layer (i.e., $\theta=1 / 3,1,3)$ are then considered, and the calculated results at 10 years are shown in Fig. 4. Comparison of the solute concentration at the same relative depth in the lower layer shows the impact of a thicker upper layer leading to significantly lower values of solute concentration with increasing total thickness (i.e., $H)$. This result is due to the increased time required to penetrate through a thicker upper layer. Overall, the results of the verification analysis indicate that the analytical simulations are in excellent agreement with the numerical simulations.

\section{Applications}

In this section, the analytical solutions presented in this paper are applied to analyze first leachate diffusion in a CCL and an underlying stratum of a landfill and, second, contaminant diffusion within a capping layer and an underlying contaminated sediment layer.

\section{Leachate Diffusion in a CCL and an Underlying Stratum}

CCLs typically have thicknesses of between 0.6 and $1.8 \mathrm{~m}$ (Benson and Daniel 1994) and a thickness of $0.9 \mathrm{~m}$ is considered herein. The soil and transport properties for the CCL considered are adopted from those presented by Lewis et al. (2009) and are listed in Table 4 together with those for the underlying stratum. A retardation factor of $R_{d}=3.3$ is adopted for the CCL following the values presented by Foose et al. (1999) for a similar material. The thickness of the underlying stratum is assumed as $1.1 \mathrm{~m}$. The leachate concentration is assumed to be constant with a value of 
Table 2. Analytical Solutions for Contaminant Diffusion in Double-Layered Soils, Scenarios with Various Combinations of BCs

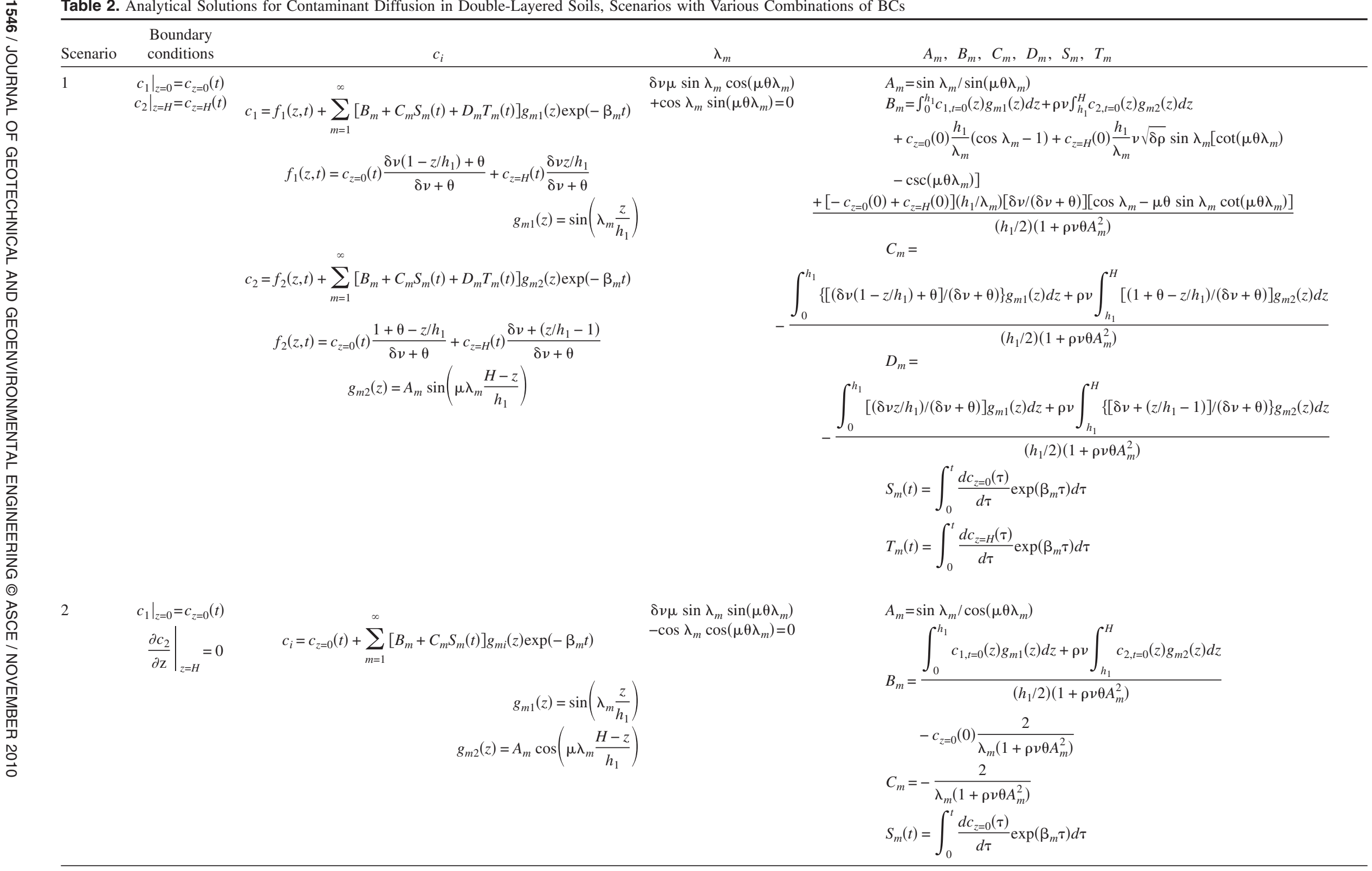


Table 2. (Continued.)

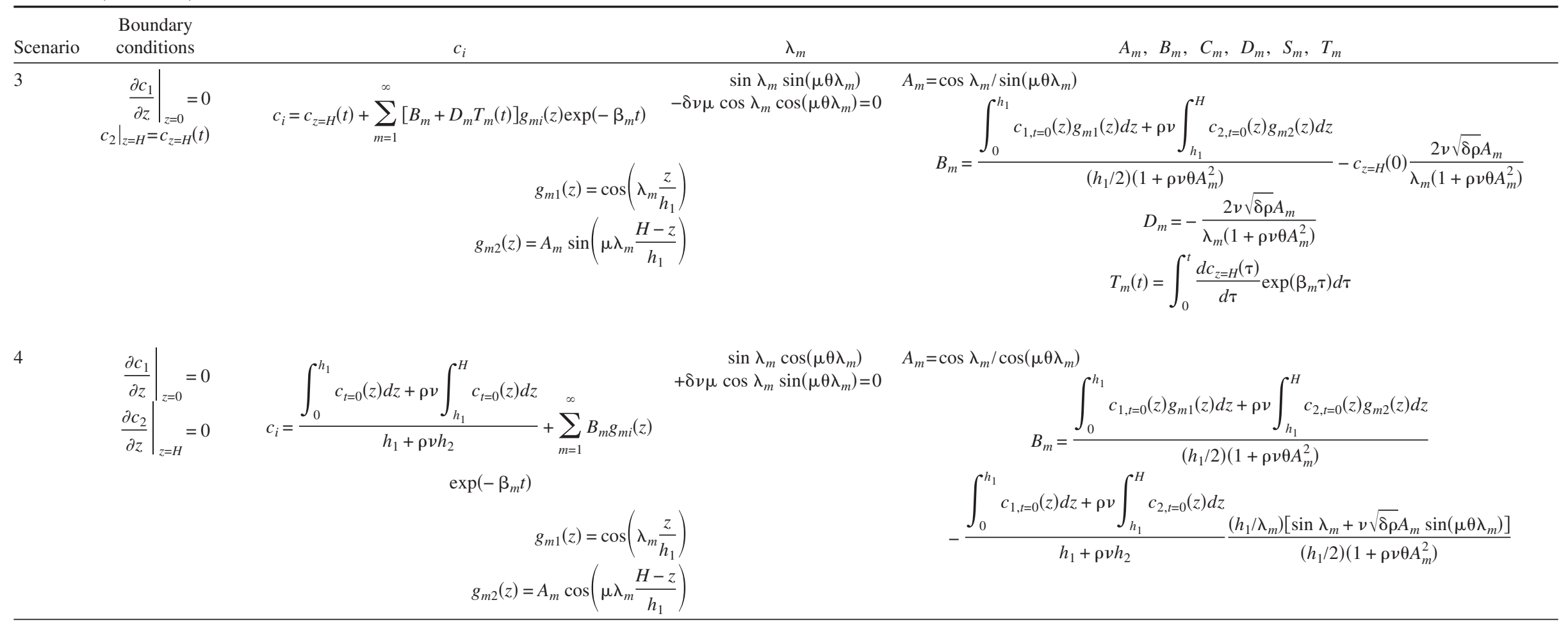

Note: $\mu=\sqrt{\rho / \delta} ; \beta_{m}=\left(D_{1}^{*} / R_{d 1}\right)\left(\lambda_{m}^{2} / h_{1}^{2}\right)$. 
Table 3. Analytical Solutions for Contaminant Diffusion in Double-Layered Soils with Particular Initial and BCs

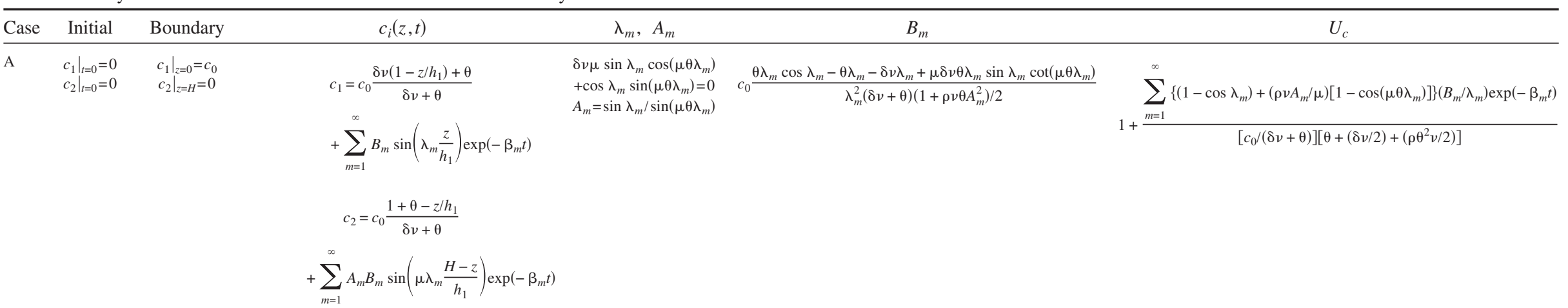

B $\quad \begin{array}{ll}\left.c_{1}\right|_{t=0}=c_{0} & \left.c_{1}\right|_{z=0}=0 \\ \left.c_{2}\right|_{t=0}=c_{0} & \left.c_{2}\right|_{z=H}=0\end{array}$

$$
\begin{gathered}
c_{1}=\sum_{m=1}^{\infty} B_{m} \sin \left(\lambda_{m} \frac{z}{h_{1}}\right) \exp \left(-\beta_{m} t\right) \\
c_{2}=\sum_{m=1}^{\infty} A_{m} B_{m} \sin \left(\mu \lambda_{m} \frac{H-z}{h_{1}}\right) \exp \left(-\beta_{m} t\right)
\end{gathered}
$$

C $\left.\quad c_{1}\right|_{t=0}=0 \quad$ As above

$\left.c_{2}\right|_{t=0}=c_{0}$

As above

$\begin{array}{cc}\left.c_{1}\right|_{t=0}=0 & \left.c_{1}\right|_{z=0}=c_{0} \\ \left.c_{2}\right|_{t=0}=0 & d c_{2} /\left.d z\right|_{z=H}=0\end{array}$

$$
\begin{array}{lc}
\left.c_{1}\right|_{t=0}=0 \\
\left.c_{2}\right|_{t=0}=0
\end{array} \quad d c_{2} /\left.d z\right|_{z=H}=0 \quad c_{1}=c_{0}+\sum_{m=1}^{\infty} B_{m} \sin \left(\lambda_{m} \frac{z}{h_{1}}\right) \exp \left(-\beta_{m} t\right)
$$$$
c_{2}=c_{0}+\sum_{m=1}^{\infty} A_{m} B_{m} \cos \left(\mu \lambda_{m} \frac{H-z}{h_{1}}\right) \exp \left(-\beta_{m} t\right)
$$

E $\left.\quad c_{1}\right|_{t=0}=\left.c_{0} \quad c_{1}\right|_{z=0}=0$ $\begin{array}{lc}\left.c_{1}\right|_{t=0}=c_{0} & \left.c_{1}\right|_{z=0}=0 \\ \left.c_{2}\right|_{t=0}=c_{0} & d c_{2} /\left.d z\right|_{z=H}=0\end{array}$

$$
c_{1}=\sum_{m=1}^{\infty} B_{m} \sin \left(\lambda_{m} \frac{z}{h_{1}}\right) \exp \left(-\beta_{m} t\right)
$$$$
c_{2}=\sum_{m=1}^{\infty} A_{m} B_{m} \cos \left(\mu \lambda_{m} \frac{H-z}{h_{1}}\right) \exp \left(-\beta_{m} t\right)
$$

As above

$$
c_{0} \frac{1+\nu \sqrt{\delta \rho} A_{m}}{\lambda_{m}\left(1+\rho \nu \theta A_{m}^{2}\right) / 2}
$$$$
c_{0} \frac{\nu \sqrt{\delta \rho} A_{m}\left[1-\cos \left(\mu \theta \lambda_{m}\right)\right]}{\lambda_{m}\left(1+\rho \nu \theta A_{m}^{2}\right) / 2}
$$

$\delta \nu \mu \sin \lambda_{m} \sin \left(\mu \theta \lambda_{m}\right)$

$-\cos \lambda_{m} \cos \left(\mu \theta \lambda_{m}\right)=0$

$A_{m}=\sin \lambda_{m} / \cos \left(\mu \theta \lambda_{m}\right)$

$$
-\frac{2 c_{0}}{\lambda_{m}\left(1+\rho \nu \theta A_{m}^{2}\right)}
$$
$\frac{2 c_{0}}{\lambda_{m}\left(1+\rho \nu \theta A_{m}^{2}\right)}$
As above

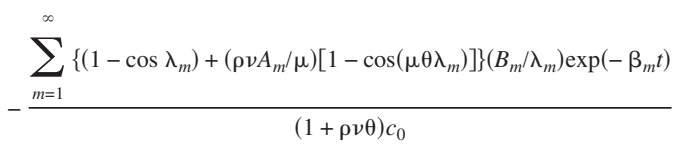

$(1+\rho \nu \theta) c_{0}$

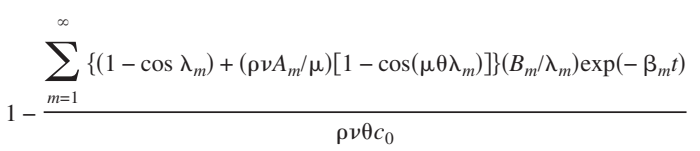

$\rho \nu \theta c_{0}$

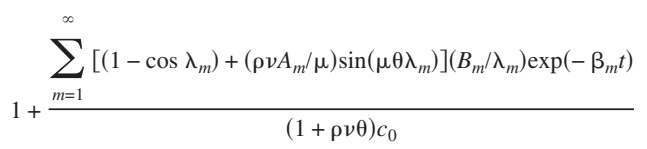

$(1+\rho \nu \theta) c_{0}$

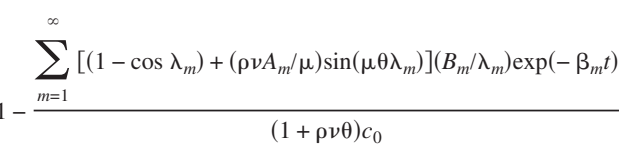


Table 3. (Continued.)

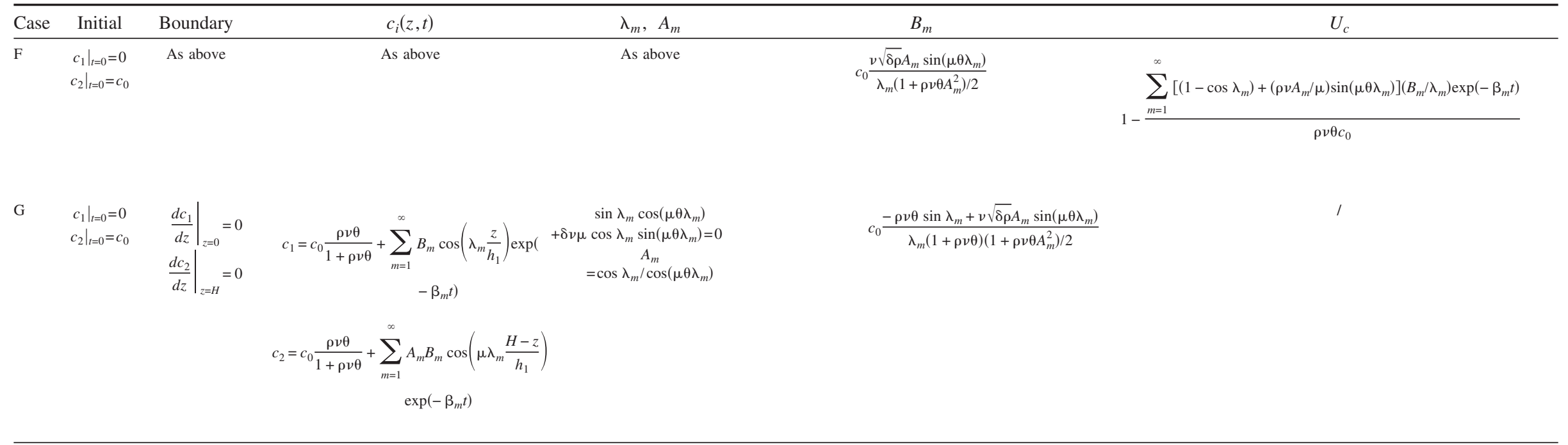

Note $\mu=\sqrt{\rho / \delta} ; \beta_{m}=\left(D_{1}^{*} / R_{d 1}\right)\left(\lambda_{m}^{2} / h_{1}^{2}\right)$. 

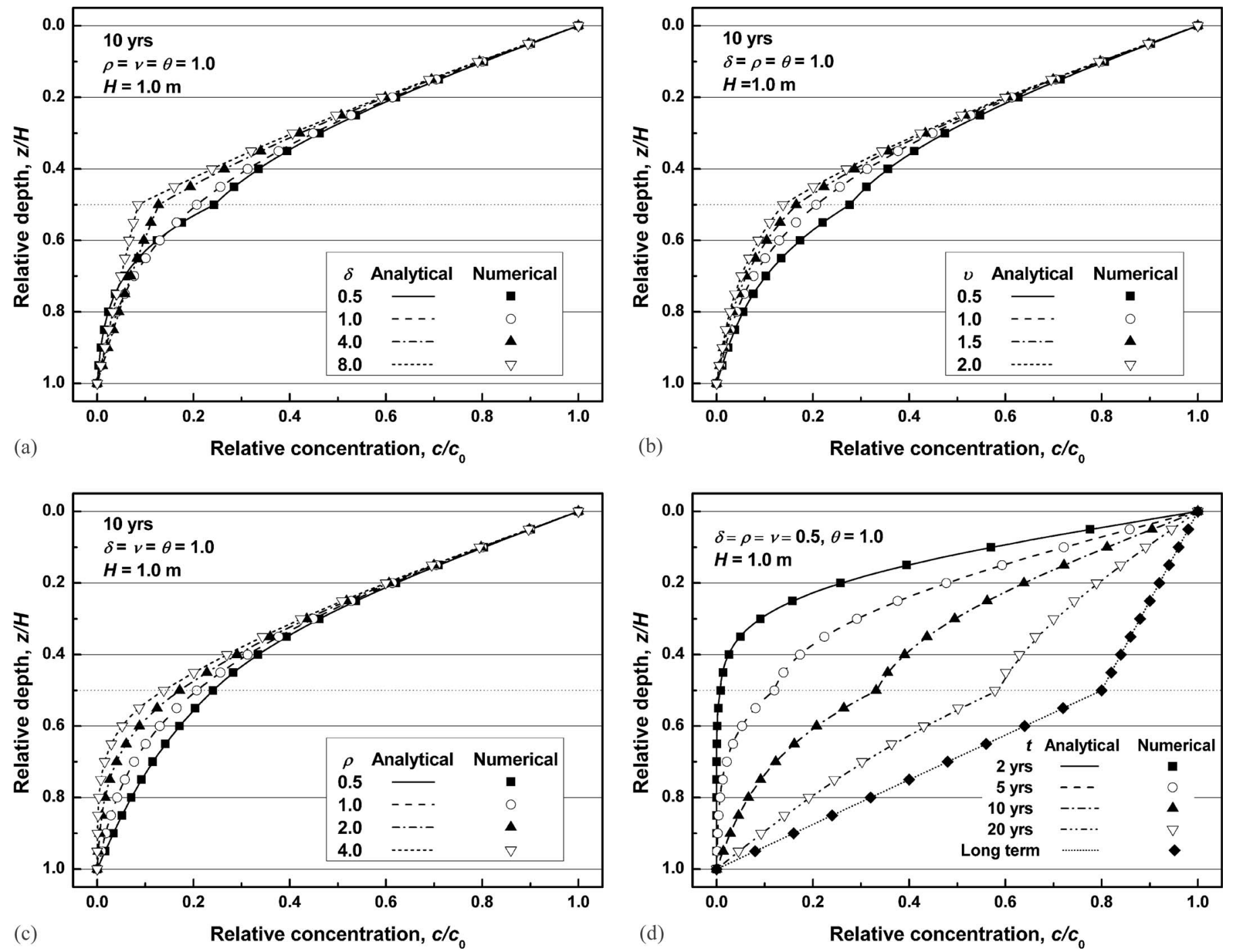

Fig. 3. Calculated solute concentration profiles with varied $\delta, \rho, v$, and $t$ for verification problem

$c_{0}$ at the top boundary and a zero concentration at the bottom boundary. This bottom BC is often considered appropriate if a confined aquifer is present below the base of the stratum, such that lateral flow through the aquifer immediately removes any contaminant mass flux entering the aquifer.

The solution for Case $\mathrm{A}$ in Table 3 is adopted to analyze leachate diffusion in the CCL and the underlying stratum according to the initial and BCs considered, and the calculated leachate concentration profiles at elapsed times of 2, 5, 10, 20, 50, and 100 years and steady state $(t=\infty)$ are shown in Fig. 5(a). Almost no leachate enters the underlying stratum before 10 years, and the concentration gradient in this layer is still relatively low after 50 years, with steady-state conditions not being achieved even after 100 years.

As shown in Fig. 5(b), the outlet leachate flux at the bottom $(H=2.0 \mathrm{~m})$, which is calculated using Eq. (6), remains close to zero during the first 40 years, and at 100 years is less than $30 \%$ of the maximum occurring after 700 years. However, without a CCL, a significant outlet leachate flux occurs at the base after 10 years, and after 100 years reaches $85 \%$ of the maximum occurring after 200 years. Also, nearly 10 years is required for the leachate to penetrate the CCL, as the flux at the interface remains low during that period. The flux at the interface has a peak value at
100 years and then drops slowly to a value being equal to that of the long-term outlet flux at the bottom, which demonstrates a mass balance for the underlying stratum after the steady-state condition is achieved.

The calculated average degrees of diffusion are also shown in Fig. 5(b) with shapes similar to those of average degrees of consolidation (Terzaghi 1943; Lee et al. 1992; Xie 1994) due to the analogy between diffusion and consolidation (Shackelford and Lee 2005). The system with a CCL has a lower average degree of diffusion than the system without a CCL due to the barrier effect of the CCL. For instance, the average degree of diffusion for the system with CCL is 0.23 at 10 years, which is less than $60 \%$ of that for the system without a CCL. Consequently, based on the concentration profiles, outlet fluxes, and average degrees of diffusion, the CCL effectively reduces leachate diffusion through the system, as expected.

\section{Contaminant Diffusion within a Capping Layer and an Underlying Contaminated Sediment Layer}

The capped contaminated sediment problem considered herein is based on the problem considered by Thoma et al. (1993). A 1.5$\mathrm{m}$-thick sediment is contaminated by trichloropropane (TCP) and 

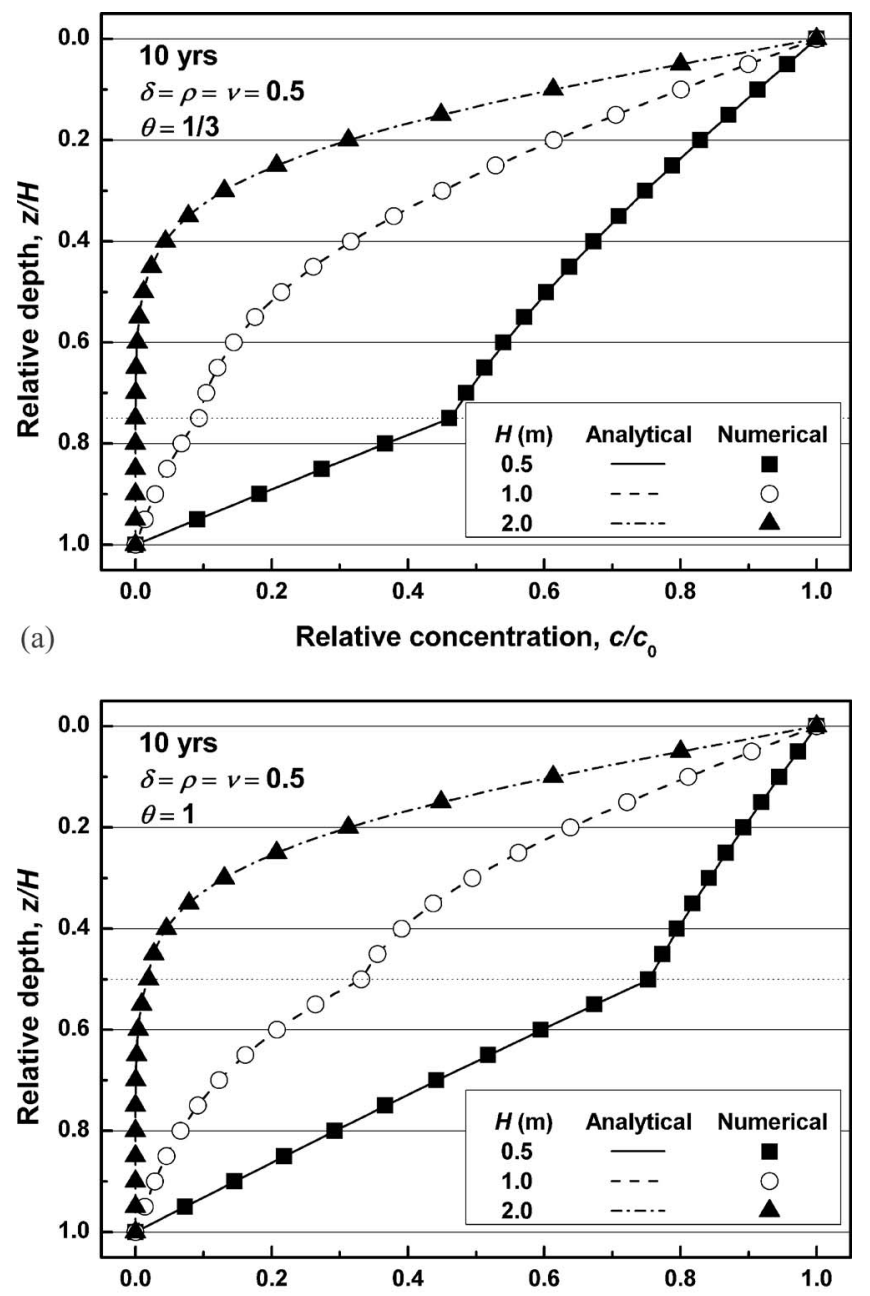

(b)

Relative concentration, $c / c_{0}$

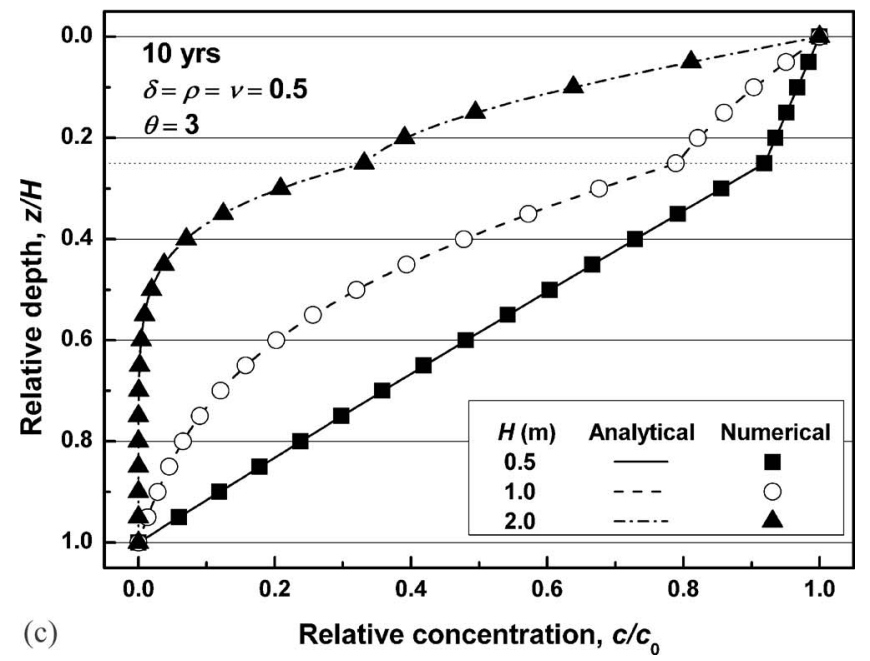

Fig. 4. Calculated solute concentration profiles with varied $\theta$ and $H$ for verification problem

covered by a $0.7-\mathrm{m}$-thick balsam sand capping layer to impede the TCP from dispersing into the surface water. The soil and transport properties considered for these two layers are as defined by Thoma et al. (1993) and are summarized in Table 5 . The initial concentration of TCP is $150 \mathrm{mg} / \mathrm{L}$ for the sediment $\left(\left.c_{s}\right|_{t=0}\right.$ $=150 \mathrm{mg} / \mathrm{L}$ ) and zero for the capping layer. A zero-flux BC is
Table 4. Soil and Transport Properties for CCL and Underlying Stratum

\begin{tabular}{lcc}
\hline Property (unit) & $\mathrm{CCL}^{\mathrm{a}}$ & Underlying stratum \\
\hline$D^{*}\left(\mathrm{~m}^{2} / \mathrm{s}\right)$ & $4 \times 10^{-10}$ & $1 \times 10^{-10}$ \\
$R_{d}$ & 3.3 & 1.0 \\
$n$ & 0.444 & 0.375 \\
$h(\mathrm{~m})$ & 0.9 & 1.1
\end{tabular}

${ }^{\mathrm{a}}$ From Lewis et al. (2009) and Foose et al. (1999).

imposed at the bottom of the sediment layer, and the concentration is fixed at zero at the top of the capping layer to model the washing effect of the surface water. This scenario matches Case F previously defined in Table 3 .

The resulting TCP concentration distributions in both the capping layer and the sediment layer at different elapsed times are shown in Fig. 6(a). In order to evaluate the impact of the capping layer on containment, a scenario without the capping layer is also considered and can be analyzed by the solution for Case E in Table 3 or the analytical solution presented by Carslaw and Jaeger (1959) and Shackelford and Lee (2005). In this analysis the sediment layer is arbitrarily separated into 0.5 - and 1.0-m-thick layers, and the soil and transport properties of the sediment are set for both of the two layers. The concentration profiles for the scenario without the capping layer are shown in Fig. 6(b). Based on
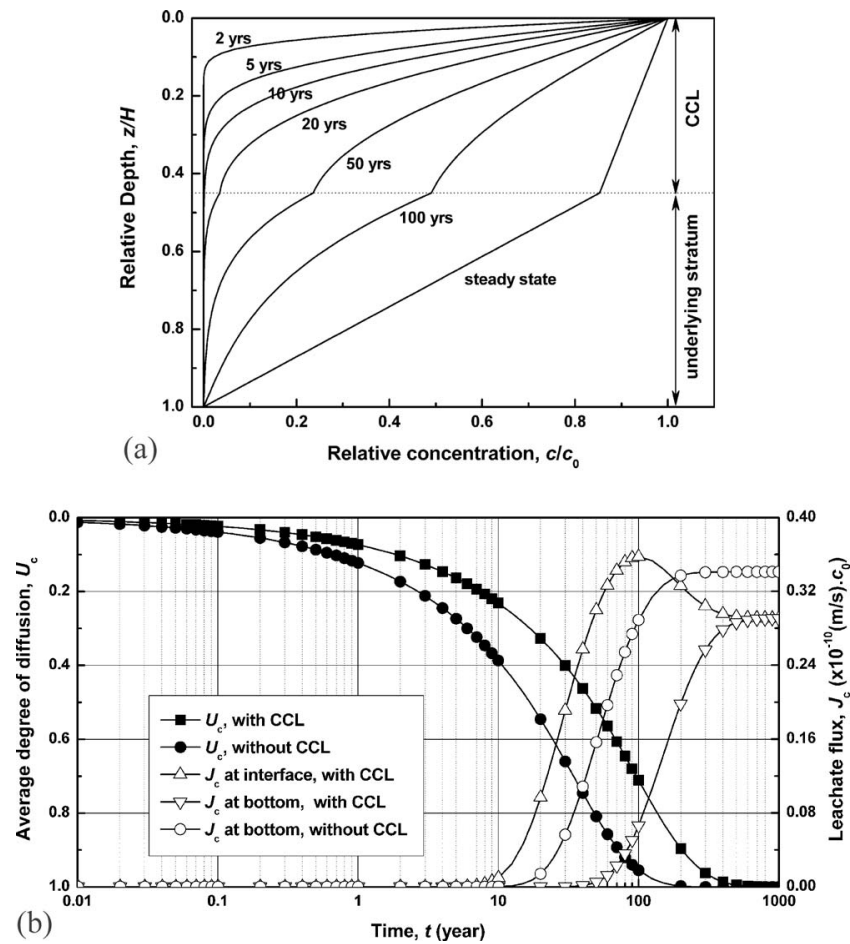

Fig. 5. Calculated leachate concentration profiles, average degree of diffusion, and leachate flux for diffusion in the CCL and the underlying stratum of a landfill

Table 5. Soil and Transport Properties for Capping Layer and Contaminated Sediment (Thoma et al. 1993)

\begin{tabular}{lcc}
\hline Property (unit) & Capping material & Sediment \\
\hline$D^{*}\left(\mathrm{~m}^{2} / \mathrm{s}\right)$ & $9.8 \times 10^{-10}$ & $9.4 \times 10^{-10}$ \\
$R_{d}$ & 4.94 & 43.3 \\
$n$ & 0.38 & 0.45 \\
$h(\mathrm{~m})$ & 0.7 & 1.5 \\
\hline
\end{tabular}



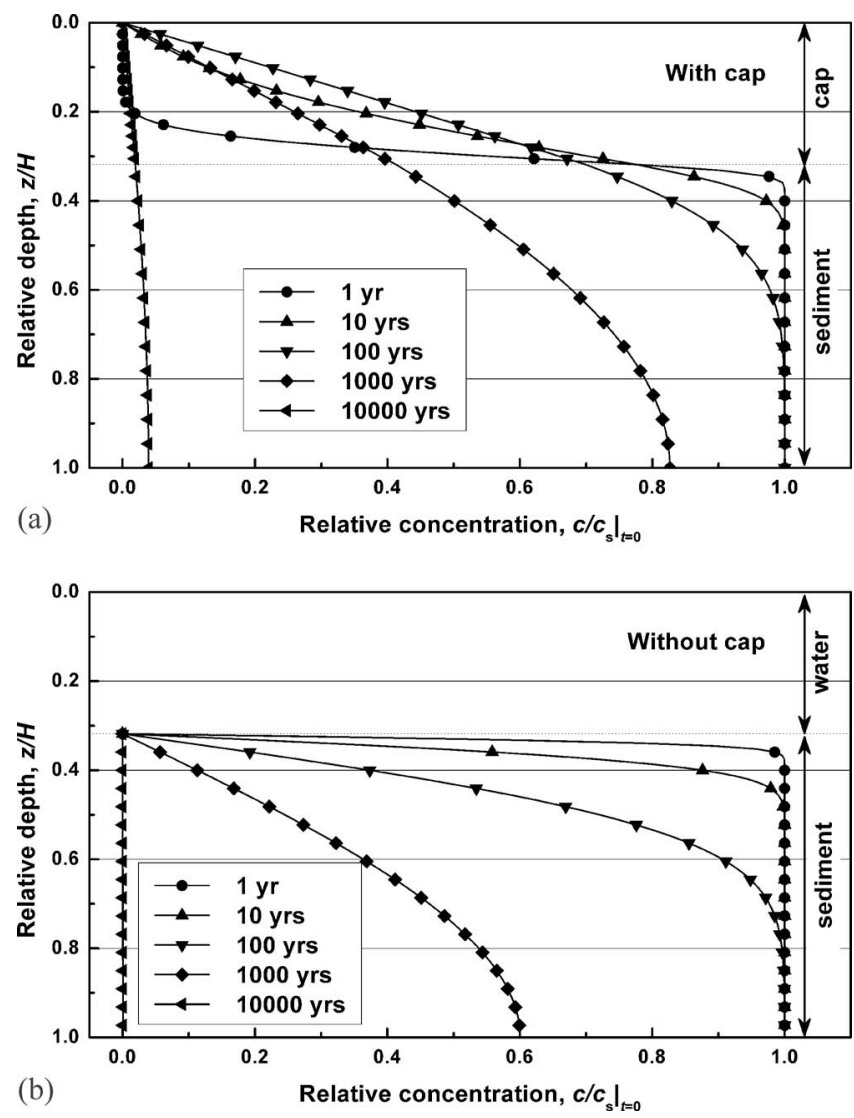

Fig. 6. Calculated TCP concentration profiles at different times for diffusion in (a) capped contaminated sediment; (b) uncapped contaminated sediment

comparison of the concentration profiles after 10 years, less TCP has been removed from the sediment for the scenario with the capping layer, as expected.
This observation can be observed more clearly in Fig. 7, where the average degree of diffusion, $U_{c}$, for both the scenarios (with and without the cap) versus time is presented. For the scenario with a capping layer $U_{c}$ is close to zero corresponding to almost no TCP diffusion into water for the first 10 years and only $3.7 \%$ of TCP has diffused into water after 100 years. However, values for $U_{c}$ of 6.2 and $20 \%$ within the first 10 and 100 years, respectively, result for the scenario without the capping layer.

For the scenarios considered above, the rate of TCP diffusion into the water is quite low, and more than 1,000 years are required to reach an average degree of diffusion of $90 \%$. This result is to a large extent caused by the high retardation factor of the sediment. To assess the impact of the retardation factor on the system, an additional analysis was performed with the retardation factor of the sediment given a significantly lower value (matching that of the capping layer). The resulting calculated average degrees of diffusion with time are also illustrated in Fig. 7. The rate of TCP diffusion is considerably greater, with average degrees of diffusion of 22 and $58 \%$ after 100 years for the scenarios with and without the cap, respectively. This result clearly shows that the retardation factor of porous media is of importance for the rate of contamination diffusion. The analyses presented herein do not include the effect of consolidation of both the sediment and underlying uncontaminated layers on the TCP transport which could result in greater TCP mass flux due to advection (Alshawabkeh et al. 2005; Arega and Hayter 2008). However, the analytical solutions presented in this paper can be regarded as a useful approach to predict the lower boundary of the rate of contaminant transport from the sediment.

The definition of average degree of diffusion used in this paper, i.e., Eq. (4), represents the relative amount of solute mass removed or gained in the double layers following the approach of Shackelford and Lee (2005). However, another definition based on solute mass in pore water presented by Chen et al. (2009) can be written for a double-layer porous medium as follows:

$$
U_{c}(t)=\frac{\int_{0}^{h_{1}} c_{1}(z, 0) d z+\int_{h_{1}}^{H} c_{2}(z, 0) d z-\int_{0}^{h_{1}} c_{1}(z, t) d z-\int_{h_{1}}^{H} c_{2}(z, t) d z}{\int_{0}^{h_{1}} c_{1}(z, 0) d z+\int_{h_{1}}^{H} c_{2}(z, 0) d z-\int_{0}^{h_{1}} c_{1}(z, \infty) d z-\int_{h_{1}}^{H} c_{2}(z, \infty) d z}
$$

Considering Case F, the average degree of diffusion based on Eq. (21) and solute mass in pore water can then be written as follows:

$U_{c}(t)=1$

$$
-\frac{\sum_{m=1}^{\infty}\left[\left(1-\cos \lambda_{m}\right)+\left(A_{m} / \mu\right) \sin \left(\mu e \lambda_{m}\right)\right]\left(B_{m} / \lambda_{m}\right) \exp \left(-\beta_{m} t\right)}{e c_{0}}
$$

The calculated average degrees of diffusion for both definitions [i.e., Eqs. (4) and (22)] are plotted versus time in Fig. 8 for the capped contaminated sediment problem considered here. An in- teresting outcome is that, when based on solute mass in the pore water, the value of average degree of diffusion is negative in the first 300 years of the analysis. This is a result of the relatively high retardation factor of the sediment compared to that of the capping layer. The solute mass diffusing from the sediment into the cap leads to a small reduction of solute concentration in the sediment, but a relatively large increase in solute concentration in the capping layer. This results in a greater average solute concentration through the double layers than that at the initial state, and subsequently according to the definition of average degree of diffusion given in Eq. (22) a negative average degree of diffusion during the early period, which is clearly problematic. However, as shown in Fig. 8, the average degree of diffusion based on solute 


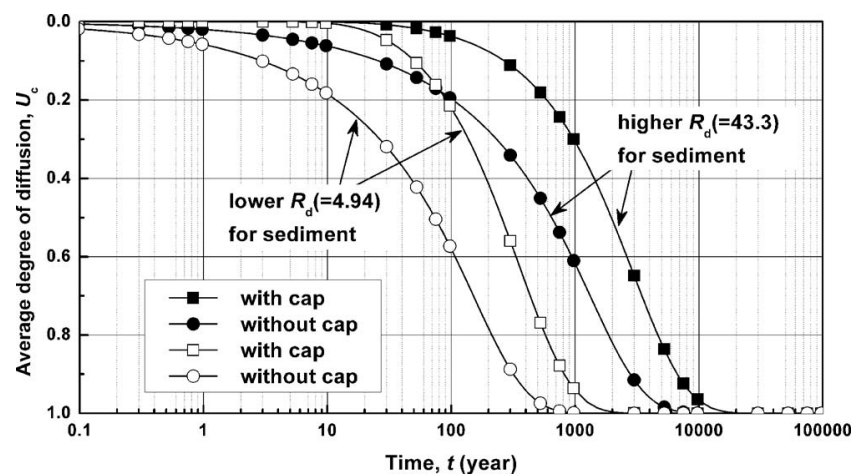

Fig. 7. Calculated average degree of diffusion with time for diffusion in a capped contaminated sediment

mass in the soil, as defined by Shackelford and Lee (2005) and applied in this paper for a two-layered system [i.e., Eq. (4)], avoids this problem as the total mass of contaminant which is conserved is considered. Consequently, the average degree of diffusion based on total contaminant mass is more reasonable than that based on solute mass in the pore water (solute concentration).

Consideration of Eq. (6) allows the following expression to be developed to calculate the TCP flux into the water:

$$
J_{c}(t)=-\frac{n_{1} D_{1}^{*}}{h_{1}} \sum_{m=1}^{\infty} \lambda_{m} B_{m} \exp \left(-\beta_{m} t\right)
$$

The calculated TCP fluxes into the water for both the scenarios with and without the capping layer, considering the original retardation factor, are shown in Fig. 9(a). The maximum flux for the scenario without the capping layer is more than 200 times greater than that for the scenario with the capping layer, and this maximum flux occurs at the start of the analysis and subsequently decreases with the time. However, the flux for the scenario with the capping layer is close to zero within the first 3 years as the capping layer prevents TCP from diffusing directly into the water. The TCP concentration then increases due to the penetration through the capping layer, reaching a maximum (i.e., 6.06 $\times 10^{-8} \mathrm{~g} / \mathrm{s} \mathrm{m}^{2}$ ) at the elapsed time of 45 years and decreases thereafter. Following the definition of breakthrough by Palermo et al. (1998a), the flux reaches $5 \%$ of its maximum value at the elapsed time of 4.25 years. These results, when considered with the average degree of diffusion shown in Fig. 7, clearly demonstrate the impact of introducing a capping layer to the system both in terms of timescales and levels of contaminant flux.

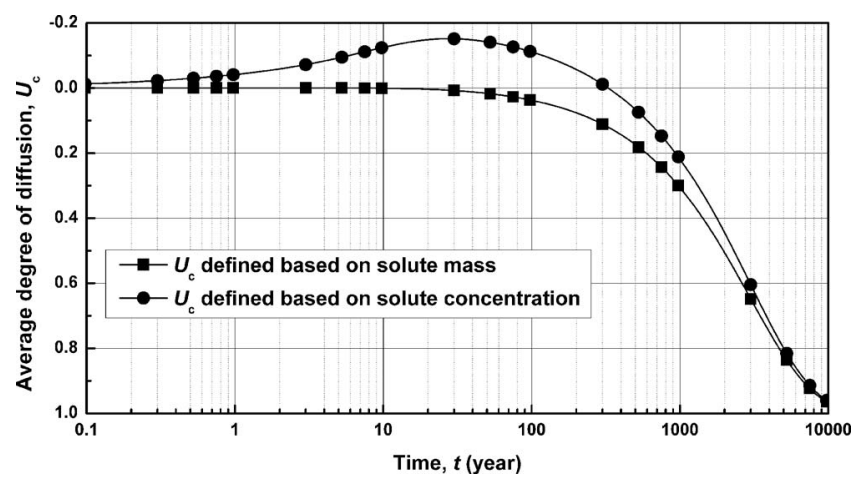

Fig. 8. Comparison of different definitions of average degree of diffusion for diffusion in a capped contaminated sediment
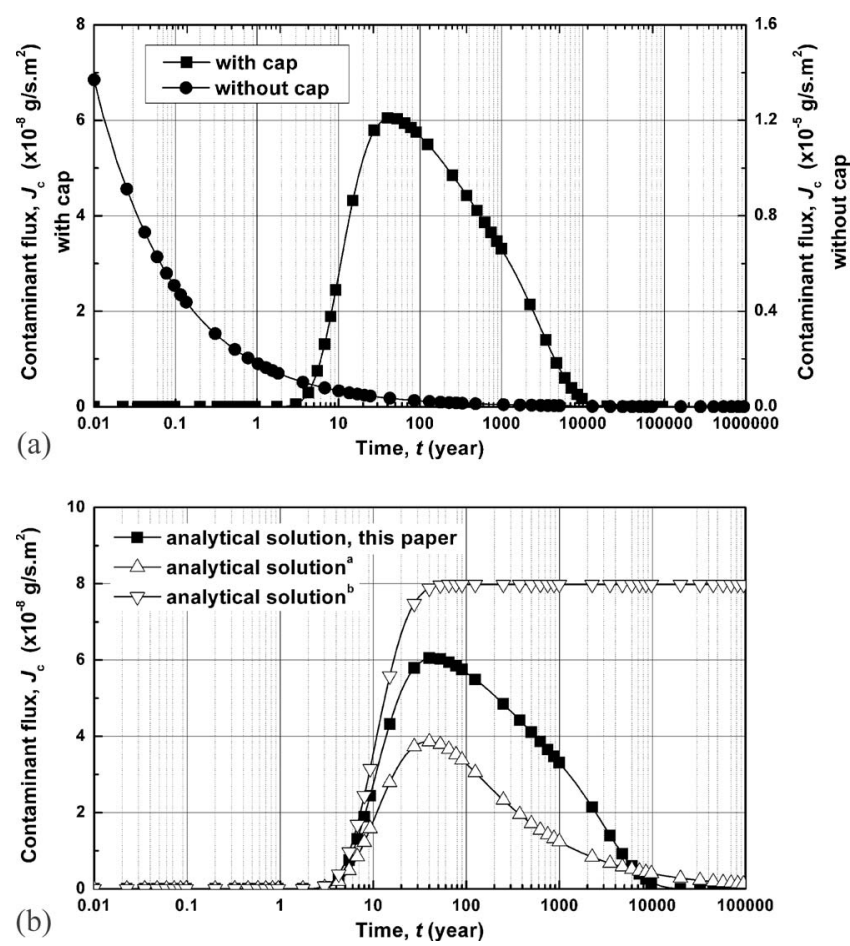

Fig. 9. Calculated TCP flux into water with time for diffusion in a capped contaminated sediment [(a) analytical solution for a finite medium; (b) analytical solution for a semiinfinite medium]

An analytical solution for diffusion in a finite homogeneous medium presented by Carslaw and Jaeger (1959) is often employed to estimate the solute diffusion in the capping layer for capping contaminated sediment (Thoma et al. 1993; Palermo et al. 1998a,b), with an assumption of constant concentration at the bottom of the capping layer. A similar analytical solution for a semiinfinite medium with the same $\mathrm{BC}$ has also been presented by Carslaw and Jaeger (1959). The solute flux from the top surface of the capping, based on these two analytical solutions for finite and semiinfinite media (Carslaw and Jaeger 1959), can be expressed as follows:

$$
\begin{aligned}
J_{c}(t)= & \frac{n_{1} D_{1}^{*} c_{0}}{h_{1}}\left[1+2 \sum_{m=1}^{\infty}(-1)^{m}\right. \\
& \left.\exp \left(-\frac{m^{2} \pi^{2} D_{1}^{*} t}{R_{d 1} h_{1}^{2}}\right)\right] \quad \text { (finite media) }
\end{aligned}
$$

$$
J_{c}(t)=n_{1} D_{1}^{*} c_{0} \sqrt{\frac{R_{d 1}}{\pi D_{1}^{*} t}} \exp \left(-\frac{R_{d 1} h_{1}^{2}}{4 D_{1}^{*} t}\right) \quad \text { (semiinfinite media) }
$$

These two alternative solutions can be used to compare the results of the solutions developed in this paper that consider the contaminated sediment layer with those that do not consider the contaminated sediment layer.

The calculated TCP fluxes into water using Eqs. (24) and (25) are illustrated in Fig. 9(b). The fluxes remain low in the first 3 years, which is in good agreement with the result calculated using the analytical solution presented in this paper. However, the TCP flux calculated by the analytical solution for a finite medium [i.e., Eq. (24)] then increases rapidly and reaches a peak value at 65 years of $7.98 \times 10^{-8} \mathrm{~g} / \mathrm{s} \mathrm{m}^{2}$, which is $31.7 \%$ more than that ob- 
tained by the analytical solution presented in this paper. The flux then remains at this peak value thereafter as a steady state, linear solute concentration distribution within the capping has been reached. The TCP flux calculated by the analytical solution for an infinite medium [i.e., Eq. (25)] has a peak value of 3.86 $\times 10^{-8} \mathrm{~g} / \mathrm{s} \mathrm{m}^{2}$ at 37.5 years, which is $36.3 \%$ less than that obtained by the analytical solution presented in this paper and decreases slowly thereafter. However, the analytical solution for an infinite medium overestimates the TCP flux in the long term (after 6,750 years), as shown in Fig. 9(b). Consequently, the solute flux from the capping layer into water may be overestimated by the analytical solution for a finite medium and underestimated by that for a semiinfinite medium, i.e., during timescales typically considered in engineering applications.

\section{Conclusions}

Analytical solutions for conservative solute diffusion in onedimensional double-layered porous media were presented in this paper. Solutions were derived for various combinations of fixed solute concentration and zero-flux BCs at the top and the bottom and for arbitrary initial solute concentration distributions throughout the media. Several solutions considering particular initial and BCs were presented based on typical contaminant transport problems found in geoenvironmental engineering. These analytical solutions were shown to correlate well with numerical solutions from a finite-element analysis.

The presented analytical solutions were used to investigate leachate transport for two typical applications: (1) a CCL from a landfill with an underlying stratum and (2) contaminant diffusion within a subaqueous capped contaminated sediment system. An alternative definition of the average degree of diffusion based on solute concentration was also considered and found to be less robust than the definition based on total solute mass adopted in this paper. Furthermore, for the capped contaminated sediment application, comparisons with alternative solutions indicated that consideration of the contaminated sediment layer is necessary to obtain reasonable estimates of contaminant fluxes from the capping layer.

\section{Acknowledgments}

The financial support received from the U.K. Engineering and Physical Sciences Research Council via Grant No. EP/C532651/2 and from the National Natural Science Foundation of China (NSFC), Grant Nos. 10972195 and 51009121 are gratefully acknowledged.

\section{References}

Alshawabkeh, A. N., Rahbar, N., and Sheahan, T. (2005). "A model for contaminant mass flux in capped sediment under consolidation." $J$. Contam. Hydrol., 78(3), 147-165.

Arega, F., and Hayter, E. (2008). "Coupled consolidation and contaminant transport model for simulating migration of contaminants through the sediment and a cap." Appl. Math. Model., 32(11), 24132428.

Benson, C. H., and Daniel, D. E. (1994). "Minimum thickness of compacted soil liners. 2. Analysis and case-histories." J. Geotech. Engrg., 120(1), 153-172.

Carslaw, H. S., and Jaeger, J. C. (1959). Conduction of heat in solids, Oxford University Press, New York.

Chapra, S. C., and Canale, R. P. (2006). Numerical methods for engineers, McGraw-Hill Higher Education, Boston.
Chen, Y., Xie, H., Ke, H., and Chen, R. (2009). "An analytical solution for one-dimensional contaminant diffusion through multi-layered system and its applications." Environ. Geol., 58(5), 1083-1094.

Cleall, P. J., Seetharam, S. C., and Thomas, H. R. (2007). "Inclusion of some aspects of chemical behavior of unsaturated soil in thermo/ hydro/chemical/mechanical models. I: Model development." J. Eng. Mech., 133(3), 338-347.

Crank, J. (1956). The mathematics of diffusion, Oxford University Press, New York.

Foose, G. J., Benson, C. H., and Edil, T. B. (1999). "Equivalency of composite geosynthetic clay liners as a barrier to volatile organic compounds." Geosynthetics 99, Industrial Fabrics Association International, Roseville, Minn., 321-334.

Freeze, R. A., and Cherry, J. A. (1979). Groundwater, Prentice-Hall, Englewood Cliffs, N.J.

Lampert, D. J., and Reible, D. (2009). "An analytical modeling approach for evaluation of capping of contaminated sediments." Soil Sediment Contam., 18(4), 470-488.

Lee, P. K. K., Xie, K. H., and Cheung, Y. K. (1992). "A study on onedimensional consolidation of layered systems." Int. J. Numer. Analyt. Meth. Geomech., 16(11), 815-831.

Leij, F. J., Dane, J. H., and van Genuchten, M. T. (1991). "Mathematicalanalysis of one-dimensional solute transport in a layered soil-profile." Soil Sci. Soc. Am. J., 55(4), 944-953.

Leij, F. J., and van Genuchten, M. T. (1995). "Approximate analytical solutions for solute transport in two-layer porous-media." Transp. Porous Media, 18(1), 65-85.

Lewis, T. W., Pivonka, P., Fityus, S. G., and Smith, D. W. (2009). "Parametric sensitivity analysis of coupled mechanical consolidation and contaminant transport through clay barriers." Comput. Geotech., 36(1-2), 31-40.

Liu, C., and Ball, W. P. (1998). "Analytical modeling of diffusion-limited contamination and decontamination in a two-layer porous medium." Adv. Water Resour., 21(4), 297-313.

Liu, C., Ball, W. P., and Ellis, J. H. (1998). "An analytical solution to the one-dimensional solute advection-dispersion equation in multi-layer porous media." Transp. Porous Media, 30(1), 25-43.

Palermo, M. R., et al. (1998a). Guidance for subaqueous dredged material capping, U.S. Army Corps of Engineers, Vicksburg, Miss.

Palermo, M. R., Maynord, S., Miller, J., and Reible, D. D. (1998b). Guidance for in-situ subaqueous capping of contaminated sediments, Great Lakes National Program Office, Chicago.

Rowe, R. K., Quigley, R. M., Brachman, R. W. I., and Booker, J. R. (2004). Barrier systems for waste disposal facilities, Taylor \& Francis, Oxford, U.K.

Seetharam, S. C., Thomas, H. R., and Cleall, P. J. (2007). "Coupled thermo/hydro/chemical/mechanical model for unsaturated soilsNumerical algorithm." Int. J. Numer. Methods Eng., 70, 1480-1511.

Shackelford, C. D. (1991). "Laboratory diffusion testing for waste disposal: A review." J. Contam. Hydrol., 7(3), 177-217.

Shackelford, C. D., and Daniel, D. E. (1991). "Diffusion in saturated soil. I: Background." J. Geotech. Geoenviron. Eng., 117(3), 467-484.

Shackelford, C. D., and Lee, J. M. (2005). "Analyzing diffusion by analogy with consolidation.” J. Geotech. Geoenviron. Eng., 131(11), 1345-1359.

Sharma, H. D., and Reddy, K. R. (2004). Geoenvironmental engineering: Site remediation, waste containment, and emerging waste management technologies, Wiley, New York.

Taylor, D. W. (1948). Fundamentals of soil mechanics, Wiley, New York. Terzaghi, K. (1943). Theoretical soil mechanics, Wiley, New York.

Thoma, G. J., Reible, D. D., Valsaraj, K. T., and Thibodeaux, L. J. (1993). "Efficiency of capping contaminated sediments in-situ. 2. Mathematics of diffusion adsorption in the capping layer." Environ. Sci. Technol., 27(12), 2412-2419.

Xie, K. H. (1994). "Theory of one dimensional consolidation of doublelayered ground and its applications." Chinese J. Geotech. Eng., 16(5), $26-38$. 